\title{
High incubation investment of females paired to attractive males in barn swallows
}

\author{
Masaru HASEGAWA ${ }^{1, \#, *}$, Emi ARAI ${ }^{2}$, Mamoru WATANABE $^{1}$ and Masahiko NAKAMURA ${ }^{2}$ \\ ${ }^{1}$ Graduate School of Life and Environmental Sciences, University of Tsukuba, 1-1-1 Tennoudai, Tsukuba-shi, \\ Ibaraki 305-8572, Japan \\ ${ }^{2}$ Laboratory of Animal Ecology, Department of Biology, Joetsu University of Education, 1 Yamayashiki-machi, \\ Joetsu-shi, Niigata 943-8512, Japan
}

\section{ORNITHOLOGICAL SCIENCE \\ (C) The Ornithological Society of Japan 2012}

\begin{abstract}
Differential parental investment is the sexual selection process in which females that have acquired an attractive male invest relatively more in his offspring than females that are paired to an unattractive male. However, it is often difficult to distinguish between differential parental investment and compensation for a decrease in parental investment by an attractive mate. Using Barn Swallows Hirundo rustica gutturalis, in which males rarely participate in incubation, we investigated differential incubation investment of females. We made the following four observations: (1) Females participate in $94 \%$ of total nest attentiveness (time that eggs were incubated). (2) Female nest attentiveness was positively correlated with the ornamentation of their mates, the size of white spots in the tail, which is a measure of male attractiveness in this population. (3) Male nest attentiveness was not related to male ornaments. (4) Total nest attentiveness was positively correlated with the size of white spots in the tail. These results are consistent with differential parental investment, but not with compensation for a decrease in parental investment by a mate. Therefore, we conclude that female Barn Swallows that have acquired an attractive male invest differentially in incubation.
\end{abstract}

Key words Hirundo rustica, Differential parental investment, Male ornaments, Parental care, Post-mating sexual selection
Differential parental investment is the sexual selection process in which females that have acquired an "attractive" male (i.e. a male which is preferred by females) invest relatively more in his offspring than females that acquired an unattractive male (Burley 1986, 1988; Møller \& Thornhill 1998; Sheldon 2000). Although this process can be subdivided into a) differential access, in which females of high reproductive value acquire attractive males, and $b$ ) differential allocation, in which females increase parental investment when paired with attractive males, both $a$ and $b$ provide males with reproductive advantages in terms of increased survivorship or quality of their young. Differential parental investment is theoretically predicted when attractive males prefer to mate with females that invest relatively more in reproduction

(Received 2 July 2011; Accepted 27 December 2011)

\# Corresponding author, E-mail: perorobomusadiobe@gmail.com

* Present address: 101, 916-3 Fujisinden, Joetsu-shi, Niigata 9430816, Japan
(Møller \& Thornhill 1998) and/or when high investment in the offspring of attractive mates provides females with higher reproductive benefits (Harris \& Uller 2009). Many studies have revealed positive relationships between female parental investment and the attractiveness of their mates, which are consistent with differential parental investment (reviewed in Møller \& Thornhill 1998; Sheldon 2000). However, positive relationships between female parental investment and the attractiveness of their mates do not necessarily validate the differential parental investment hypothesis.

The positive relationships may also be explained in several other ways (Sheldon 2000). First, attractive males may provide females with a favorable environment for reproduction. For example, attractive males may have food-rich territories, which enable their mates to invest more energy, if these males also receive an advantage from holding good territories. Second, high parental investment of females paired 


\section{HASEGAWA et al.}

with attractive males may reflect compensation for low parental investment by her attractive mate rather than a decision to invest more based on the perceived quality of the mate alone (e.g. Witte 1995; Sanz 2001). There could be a tradeoff between caring for young and gaining (extra-pair) matings with different females, and it might be advantageous for attractive males to increase their chances of such matings by devoting less effort to their young than would unattractive males (e.g. Mitchell et al. 2007; reviewed in Magrath \& Komdeur 2003; Houston et al. 2005). This is possible even under experimental manipulation of male attractiveness, if males receive cues about their altered phenotypic attractiveness from their mates or neighboring individuals (see discussion in Johnsen et al. 2005). In addition, experimental manipulation itself may handicap individuals thereby reducing their parental investment, which is compensated for by their mate (Sheldon 2000).

One good opportunity to study differential parental investment, involves focusing on parental investments to which males rarely contribute, where compensation is not confounded with differential parental investment. One such example is maternal investment in eggs in birds, because males only contribute their sperm to eggs, thus it is possible to decouple differential parental investment from compensation (e.g. androgen levels in eggs; Safran et al. 2008; egg volume or mass; reviewed in Horváthová et al. 2011). Likewise, even after egg laying, female parental investment (such as incubation, brooding, and feeding young) can be studied without confounding the effects with compensation, when males rarely contribute to parental care (reviewed in Møller \& Thornhill 1998).

Incubation behavior of certain bird species, wherein males rarely contribute to incubation, offers opportunities to study differential parental investment. Differential incubation investment of females is rarely studied, even when including species where males also incubate (e.g. Václav \& Hoi 2002; Gorman et al. 2005), probably because it is less intuitive how differences in incubation behavior impact the fitness of caregivers and their offspring compared with other aspects of parental investment such as egg quality and feeding effort. However, incubation behavior is in fact an energetically demanding behavior (Tinbergen \& Williams 2002) and variation in incubation investment can have important consequences for fitness (e.g. Gorman \& Nager 2004; Gorman et al. 2005). Thus, differential incubation investment, if any exists, would exert important fitness consequences on females, which in turn has fitness consequences for males. Since females are known to compensate their own investment in incubation in response to the reduced incubation effort of attractive males, at least in the laboratory (Gorman et al. 2005), using species with males that rarely contribute to incubation will provide better understanding about differential incubation investment.

The Barn Swallow Hirundo rustica is a model species of sexual selection (Møller 1994; Turner 2006), and has multiple male ornaments, such as long outer tail feathers (Møller 1988), ventral plumage coloration including a red throat patch (Ninni 2003; Safran \& McGraw 2004), and white spots in the tail (Kose \& Møller 1999; Kose et al. 1999). Since males do not defend feeding territories, but do defend small territories containing old nests (Turner 2006), the influence on territory quality on female behavior might be expected to be small. In addition, since males rarely contribute to incubation (females incubate alone in Europe, but males incubate for $9 \%$ of the total amount of incubation during daylight hours in North America; Smith \& Montgomerie 1992) and only females can perform efficient incubation in this species (Voss et al. 2008), it is unlikely that high female incubation investment reflects compensation for low incubation investment by its mate. Using the North American subspecies H. r. erythrogaster, Smith and Montgomerie (1992) have investigated, and find no clear relationship between female incubation investment and male tail length, a measure of male attractiveness (see also Møller 1994). However, it is unclear whether the results actually indicate that there is no differential parental investment. Because previous studies in North America have shown that tail length is not closely linked to male attractiveness (e.g. Safran \& McGraw 2004; Neuman et al. 2007), females may exhibit differential parental investment based on other traits, such as plumage coloration (Safran \& McGraw 2004).

Here, studying the Barn Swallow H. r. gutturalis in Asia, we investigated differential incubation investment of females. To date, only a little information about incubation behavior of this subspecies has been published (e.g. Nakamura \& Nakamura 1993; Kojima et al. 2009). Thus, first we described detailed incubation behavior in this subspecies. Then, we investigated the relationship between female nest attentiveness (the total time that females incubated their eggs per two hours) and male ornamentation. Since the 
extent of white spots in the tail has been suggested to be a measure of male attractiveness in $H$. r. gutturalis (mating pattern; Hasegawa et al. 2010; within-pair paternity; Hasegawa et al. unpublished data), we predicted that females that acquired a male with large white tail spots should incubate more than other females. We also investigated the relationship between female incubation investment and three other male ornaments: tail length, throat patch size, and throat coloration, to exclude the possibility that it was not white tail spots, but other traits, that were subject to differential parental investment. Finally, we also studied the consequence of differential incubation investment on the incubation period that is predicted for this species (Voss et al. 2008).

\section{MATERIALS AND METHODS}

This study was conducted in 2006 in a residential area of Joetsu City, Niigata Prefecture, Japan $\left(37^{\circ} 07^{\prime} \mathrm{N}, 138^{\circ} 15^{\prime} \mathrm{E}\right)$. Swallows nest there under the eaves of a covered sidewalk along the street and breed in a loose colony (see Tajima \& Nakamura 2003). We inspected nests every other day to record breeding events. This allowed the determination of laying date (which was expressed as the date of laying the first egg of the first clutch), and clutch size. We inspected nests every day around the estimated hatching date (10 days after incubation).

\section{1) Capture and measurement of birds}

Adults were captured in sweep nets while roosting at night. Birds were provided with a numbered aluminium ring and an individual combination of two or three half-sized coloured plastic rings, which was made from plastic rings (AC Hughes, Middlesex). The sex of an individual was determined by the presence (female) or absence (male) of an incubation patch. Adults were placed into two groups: ASY (after second year) birds, and age-unknown birds, based on ringing records.

At capture, we also measured tail length, the sizes of the white spots in the tail, the size of the throat patch, and collected some throat feathers. We also measured body mass, which is often used as a body condition index in this species (e.g. Dreiss et al. 2008). Although female mass would be expected to change during the breeding cycle, we found no significant relationships between female body mass and capture date $(n=21$, Spearman's rank correlation, $\left.r_{s}=0.19, P=0.39\right)$ and between female body mass and the number of days passed from laying date to capture date $\left(\mathrm{n}=21, \mathrm{r}_{\mathrm{s}}=-0.09, \mathrm{P}=0.69\right)$. Thus, we used a raw body mass value as a condition index. Tail length was estimated as the right outermost tail feather to the nearest $0.01 \mathrm{~mm}$. The size of the white spots in the tail was defined as the sum of the length of the white spots of the two outermost tail feathers on the right side. This method differs from those of previous studies (Kose \& Møller 1999; Kose et al. 1999), but it was chosen to minimize the handling time of the birds (cf. Hasegawa et al. 2010).

The throat patch area was defined as the area of the swallow's red throat patch. We put a transparent plastic sheet on the throat region ensuring that the feathers lay flat in their natural position and traced the size of the patch on the sheet with a marker pen (cf., Lendvai et al. 2004). We scanned the sheet and measured the area of the patch $\left(\mathrm{mm}^{2}\right)$ using Scion Image software (Scion Corporation, Frederick, MD). Each bird's throat patch was traced twice. We used the means of these two measurements (cf. Hasegawa et al. 2010).

Once in the laboratory, we piled five feathers on a piece of white paper, so that the perimeters of the feathers coincided. The feather samples were scanned at 800-dpi resolution, using a scanner (GT 9300 UF; Epson, Tokyo, Japan), and the images obtained were imported into Photoshop Elements 3.0 (Adobe Systems, San Jose, CA). We measured their mean redgreen-blue $(\mathrm{RGB})$ values in a square of $30 \times 30$ pixels near the distal end of the feather sample. The mean RGB values were converted into hue-saturationbrightness (HSB) values, using the algorithm described in Foley and Van Dam (1984). The repeatability of these variables was highly significant $(0.65 \leq$ repeatability $\leq 0.91, \mathrm{n}>30, \mathrm{~F}>4.69, \mathrm{P}<0.001$; see Hasegawa et al. 2008). The detailed methodology is described elsewhere (Hasegawa et al. 2008). Since the hue and brightness of throat coloration fades linearly with time, we used saturation value as a representative of throat coloration (Hasegawa et al. 2008; Hasegawa et al. 2010).

\section{2) Observing incubation}

Nests were watched only when the birds were easy to identify. Video camera recorders (SONY CCDTRV92) were set up circa $3 \mathrm{~m}$ from the nests and did not seem to disturb the birds. We only studied first nesting attempts. Each nest was observed for 120 minutes only once, either four, eight or twelve days into the incubation period. Since swallows incubate 
M. HASEGAWA et al.

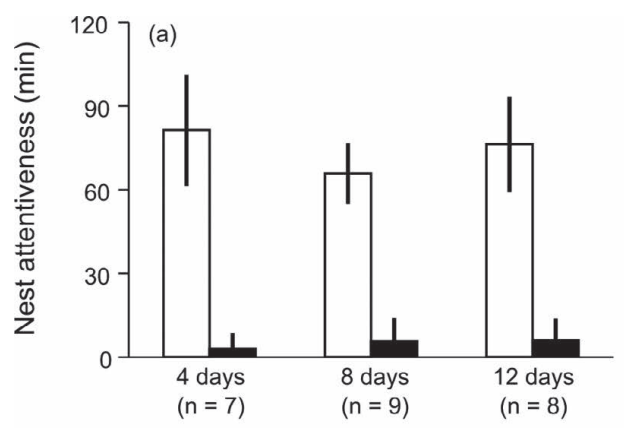

Days after begining incubation

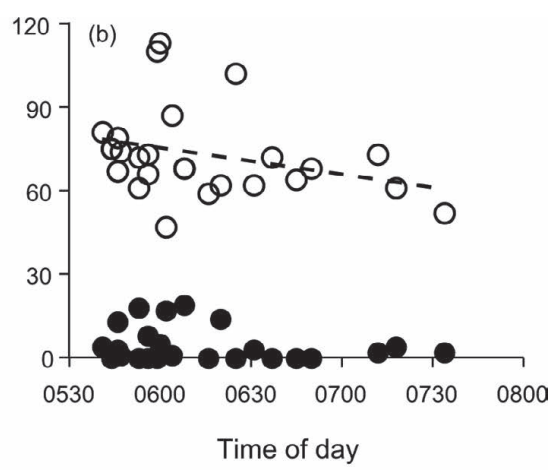

Fig. 1. Relationships between nest attentiveness and breeding stage (a) and time of day (b): male nest attentiveness (closed bars or circles), female nest attentiveness (open bars or circles). Error bars depict \pm 1 SD. Simple regression line is shown.

for approximately 14 days in order to hatch their nestlings (Turner 2006), our video recordings were made during the early, middle, and late period of incubation. We recorded male, female, and total nest attentiveness (i.e. the total time each bird incubated the clutch per $120 \mathrm{~min}$ ). We also recorded the mean length of each incubation bout and the frequency of incubation bouts within $120 \mathrm{~min}$. Observations were all made during mornings (0530-0800; Fig. 1).

\section{3) Statistical analyses}

When analyzing male attractiveness and incubation behavior, we used nest attentiveness as this is the most widely used measure of incubation behavior (e.g. Smith \& Montgomerie 1992). We first analyzed the general incubation pattern by using non-parametric methods (e.g. Mann-Whitney). Then, nest attentiveness was analyzed using a generalized linear mixed model (GLMM; lmer in R package lme4) with a quasi-binomial error structure and a logit link function. By using R's cbind function, a 2-column matrix of 'nest attentiveness' and 'absent time' (i.e. the bird did not incubate the clutch: 120 - nest attentiveness) was used as the response variable. In the models, breeding stage was considered as a random factor to control for the difference in the breeding stage we observed. The significance of the terms in a GLMM was based on the difference in deviance and degrees of freedom of the models with and without the predictor in question. In each analysis, we fitted a full model containing all explanatory variables and covariates that were supposed to have an influence on nest attentiveness. Since a measurement of throat coloration was lacking for one male $(1 / 24)$, we excluded that male from the analysis of the relationship between nest attentiveness and male ornamentation.
However, that exclusion did not affect the results. A final model was selected by progressively eliminating non-significant explanatory variables $(\mathrm{P}>0.05)$. To exclude the possibility that, high female incubation investment reflects compensation for their mates, whatever small effort males contribute (see Results), we also investigated the relationship of male nest attentiveness and total nest attentiveness of pairs in relation to male ornamentation. If high parental investment of females paired with attractive mates may reflect a decision to invest more, based on the perceived quality of the mate, rather than compensation for a decrease in parental investment by its attractive mate, then total nest attentiveness, but not male nest attentiveness, should be positively related to male ornamentation. All predictor variables were standardized to a mean of zero and a variance of one as in standardized selection analysis (Lande \& Arnold 1983). In the analysis of the incubation period (i.e. the time between laying of the last egg and hatching date), we used Spearman's partial rank correlation coefficient to control for the difference in breeding stages, because the dependent variable had only four categories (see Results), which does not fit GLMM with a normal distribution. The mean values of all statistical measurements are reported with \pm 1 SD. All data analyses were performed using the R (version 2.8.0) statistical package (R Development Core Team 2008).

\section{RESULTS}

\section{1) General incubation pattern}

Total nest attentiveness was $77.58 \pm 15.26$ minutes (range $=54-118, \mathrm{n}=24$ ) per two hours. The proportion of incubation by males was $6.20 \pm 8.43 \%$ 
(range $=0-26.56)$. Female nest attentiveness $(72.83 \pm$ $16.38, \mathrm{n}=24$, range $=47-113$ ) was significantly longer than that of males $(4.75 \pm 6.41, \mathrm{n}=24$, range $=0$ 19; Mann-Whitney U-test, $\mathrm{z}=5.94, \mathrm{P}<0.0001$ ). Among 24 pairs, approximately 40 percent of males did not incubate at all (females: 0 out of 24, males: 9 out of 24; $\mathrm{P}<0.01$, Fisher's exact test). In addition, compared to males, females undertook longer incubation bouts (females: $13.12 \min \pm 11.42 ; \mathrm{n}=24$, range $=4-56.5$; males: $5.09 \min \pm 5.47, \mathrm{n}=15$, range $=1-18 ; \mathrm{z}=3.58, \mathrm{P}<0.0001)$ and more incubation bouts (females: $7.83 \pm 3.58, \mathrm{n}=24$, range $=2-14$; males: $1.08 \pm 1.21, \mathrm{n}=24$, range $=0-4, \mathrm{z}=5.71$, $\mathrm{P}<0.0001$ ). Nest attentiveness was negatively, but not significantly, correlated within pairs (Spearman's rank correlation, $r_{s}=-0.34, \mathrm{P}=0.11$ ), indicating that compensation by one mate for a decrease in parental investment by the other mate is at best small.

Neither total nest attentiveness, nor female or male nest attentiveness, varied systematically during the incubation period $(\mathrm{n}=24$, Kruskal-Wallis test, $\chi^{2}<3.95, \mathrm{P}>0.14$; Fig. 1a). Similarly, neither total nest attentiveness, nor female and male nest attentiveness was significantly related to laying date (Spearman's rank correlation, $\left|\mathrm{r}_{\mathrm{s}}\right|<0.19, \mathrm{P}>0.37$ ) and observation date $\left(\left|\mathrm{r}_{\mathrm{s}}\right|<0.20, \mathrm{P}>0.20\right)$. On the other hand, time of day was negatively correlated with total nest attentiveness $\left(n=24, r_{s}=-0.46, P=0.02\right)$ and female nest attentiveness $\left(r_{s}=-0.39, P=0.057\right.$; Fig. 1b). Although time of day was not significantly related to male nest attentiveness $\left(r_{s}=-0.15, P=0.48\right.$; Fig. 1b), male nest attentiveness also tended to decrease with time (Spearman's partial rank correlation $\mathrm{r}_{\mathrm{sp}}=-0.33, \mathrm{P}=0.11$ ) after controlling for female nest attentiveness which was weakly negatively related to male nest attentiveness (see above).

Neither total nest attentiveness, nor female or male nest attentiveness was significantly related to clutch size $\left(\right.$ mean $=5.17$, range $=4-6, \mathrm{n}=24,\left|\mathrm{r}_{\mathrm{s}}\right|<0.10$, $\mathrm{P}>0.64)$, female body mass $\left(\mathrm{n}=21,\left|\mathrm{r}_{\mathrm{s}}\right|<0.12\right.$, $\mathrm{P}>0.59)$, nor female age $(\mathrm{n}=21$, Mann-Whitney U-test, $\mathrm{z}<1.17, \mathrm{P}>0.24)$. Similarly, neither total nest attentiveness, nor female or male nest attentiveness, was significantly related to male body mass $(n=23$, $\left.\left|\mathrm{r}_{\mathrm{s}}\right|<0.13, \mathrm{P}=0.54\right)$, or male age $(\mathrm{n}=23$, MannWhitney U-test, $\mathrm{z}<0.25, \mathrm{P}>0.81)$.

\section{2) Male ornaments and nest attentiveness}

Female nest attentiveness was significantly related to three variables: the size of the male's white tail spots, time of day, and the mate's attentiveness (Table 1). Female nest attentiveness increased with increasing size of their mate's white tail spots (Fig. 2), and decreased with passing time of day and increasing mate's attentiveness (Table 1).

Male nest attentiveness was significantly related to two variables: time of day, and mate's nest attentiveness (Table 1). Male nest attentiveness decreased

Table 1. GLMM models analyzing female nest attentiveness, male nest attentiveness, and total nest attentiveness in relation to male ornaments $(n=23)$.

\begin{tabular}{lccc}
\hline & $\begin{array}{c}\text { Female } \\
\text { attentiveness }\end{array}$ & $\begin{array}{c}\text { Male } \\
\text { attentiveness }\end{array}$ & $\begin{array}{c}\text { Total } \\
\text { attentiveness }\end{array}$ \\
\hline Explanatory variables & & & \\
$\quad$ Size of white tail spots & $\dagger 0.28(<0.0001)$ & $-0.20(0.21)$ & $\dagger 0.25(<0.0001)$ \\
Tail length & $0.02(0.62)$ & $0.02(0.87)$ & $0.00(0.92)$ \\
Throat patch area & $0.00(0.98)$ & $0.02(0.83)$ & $0.00(0.93)$ \\
Throat coloration & $0.07(0.07)$ & $-0.17(0.15)$ & $0.06(0.13)$ \\
Covariates & & & - \\
$\quad$ Mate's attentiveness & $\dagger-0.11(0.03)$ & $\dagger-0.95(<0.0001)$ & - \\
Time of day & $\dagger-0.16(<0.001)$ & $\dagger-0.90(<0.0001)$ & $\dagger-0.22(<0.0001)$ \\
\hline
\end{tabular}

Separate models were run for each column, which represents the results of GLMMs with quasi-binomial distribution with logit link function: estimates of each coefficient of each variable. Random factor is the breeding stage (4, 8, 12 days after the start of incubation). $\mathrm{P}$ values, based on $\chi^{2}$ test, are shown in parentheses. Coefficients and $\mathrm{P}$ values of significant terms $(\mathrm{P}<0.05)$ are from the final model, whereas coefficients and $\mathrm{P}$-values of non-significant terms are from the final model and each nonsignificant term added separately.

$\dagger$ indicates the variables retained in the final models. 


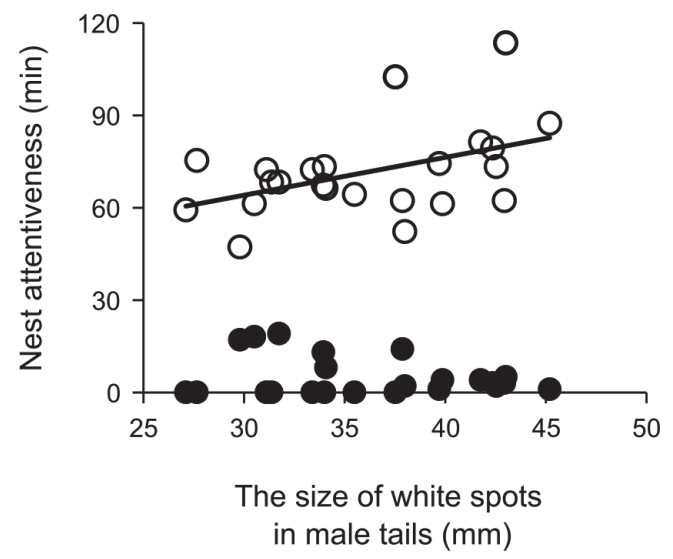

Fig. 2. Male (closed circles) and female (open circles) nest attentiveness in relation to the size of white spots in the male's tail. Simple regression line is shown.

with passing time of day and increasing mate's attentiveness. Neither the size of the male's white tail spots, nor the three other male ornaments were significantly related to male nest attentiveness (Table 1).

Total nest attentiveness was significantly related to two variables, the size of white tail spots in males and time of day (Table 1). It increased as the size of white tail spots in males increased, and decreased with increasing time of day (Table 1).

\section{3) Nest attentiveness and incubation period}

For successfully hatched clutches, the mean incubation period was 15.23 days $(n=17$, range $=14-17)$. After controlling for breeding stage, female nest attentiveness was negatively correlated with incubation period $(\mathrm{n}=17$, Spearman's partial rank correlation coefficient, $\mathrm{r}_{\mathrm{sp}}=-0.50, \mathrm{P}=0.03$; Fig. 3), indicat-

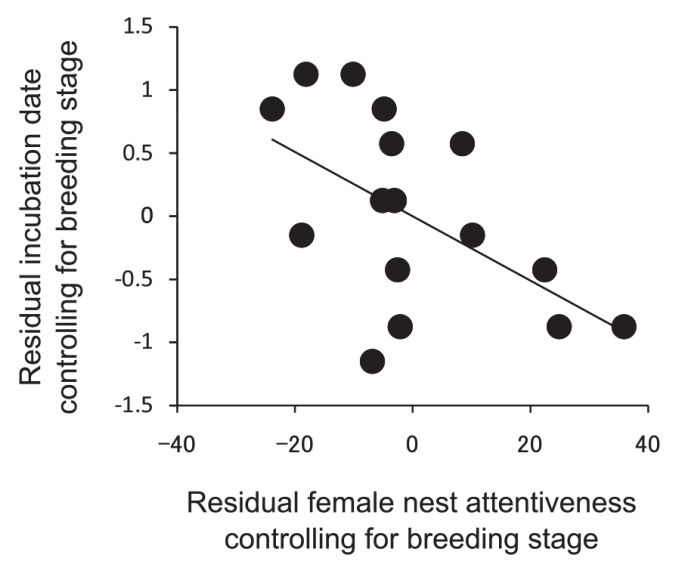

Fig. 3. Relationship between female nest attentiveness and incubation period after statistically controlling for breeding stage. Simple regression line is shown. ing that the high nest attentiveness of females brought the hatching date of clutches earlier. Incubation period was not significantly related to male nest attentiveness $\left(\mathrm{n}=17, \mathrm{r}_{\mathrm{sp}}=0.42, \mathrm{P}=0.08\right)$, total nest attentiveness $\left(\mathrm{n}=17, \mathrm{r}_{\mathrm{sp}}=-0.35, \mathrm{P}=0.16\right)$, or clutch size $\left(\mathrm{n}=17, \mathrm{r}_{\mathrm{sp}}=-0.22, \mathrm{P}=0.39\right)$.

\section{DISCUSSION}

\section{1) General incubation pattern}

Male and female Barn Swallows in Asia (H. $r$. gutturalis) participate in incubation (Fig. 1). This is consistent with previous studies that also reported male incubation in the Asian subspecies (e.g. Nakamura \& Nakamura 1993; Kojima et al. 2009). However, male Barn Swallows contributed only $6 \%$ of the total amount of incubation. In addition, males incubate more often early in the morning than at other times of day (Smith \& Montgomerie 1992). As the morning period was the one we studied, the relative contribution of males throughout the daylight period must be much smaller than the figure indicated, and thus males are at best considered to make only a small contribution to incubation.

\section{2) Male ornaments and nest attentiveness}

The current study is consistent with differential parental investment by females (Møller \& Thronhill 1998), because females that have acquired an attractive male, measured by the size of white spots in the tail (e.g. Hasegawa et al. 2010; Hasegawa et al. in preparation) incubated more than did other females (Fig. 2). The probability that high parental investment by a female paired with an attractive male may reflect compensation for a decrease in parental investment by its attractive mate (e.g. Witte 1995; Sanz 2001), is considered to be small. First, as stated above, male contribution to incubation was only small (c 6\%). Second, male incubation investment was not correlated with a measure of his attractiveness, i.e. the size of his white tail spots (Table 1). Third, even after controlling for male incubation effort, females that acquired a mate with large white tail spots incubated more than did other females (Table 1). Fourth, total nest attentiveness was positively correlated with the size of male tail spots (Table 1). Therefore, high parental investment by a female may reflect her decision to invest based on the perceivable quality of her mate.

These results are also consistent with previous studies in this species. Using white spots in the tail, 
the same measure of attractiveness as in the current study, Kose and his colleagues have shown that experimentally reducing spot area led to reduced male reproductive success in European Barn Swallow populations (Kose \& Møller 1999; Kose et al. 1999), suggesting differential investment by females in reproduction when mated to a particularly attractive male. In addition, there have also been some studies that have shown that females basing pairing on some measure of attractiveness (e.g. tail length, or plumage coloration), invest more in reproduction (e.g. feeding rates, brood number; de Lope \& Møller 1993; Møller 1994; androgen levels in eggs: Safran et al. 2008). Smith and Montgomerie (1992) studied differential parental investment at incubation. However, they could not find a clear relationship between male tail length (a measure of male attractiveness), and incubation behavior (Smith \& Montgomerie 1992), although whether tail length could be used as a measure of male attractiveness was not certain (e.g. Safran \& McGraw 2004). To our knowledge, the current study is the first to confirm differential incubation investment by female Barn Swallows, a species in which males rarely contribute to incubation.

Although previous studies on differential parental investment have rarely focused on incubation behavior, differential parental investment in incubation would be expected to exist as we have found here. Incubation behavior differs from other kinds of parental care (e.g. feeding efforts) in that males cannot compensate perfectly, because they lack a brood patch, which is necessary for effective incubation (Voss et al. 2008). In fact, we found that it was only female nest attentiveness that helped bring forward the incubation date, suggesting that male incubation is not effective. In this point, differential incubation effort is similar to maternal investment in eggs, which males cannot contribute to other than with their sperm. Less-attractive males would thus just undertake extended incubation (Fig. 3), even if they noticed that their mate was a poor caregiver. It remains to be determined whether these males have some other alternative strategies to increase reproductive success (i.e. increasing other elements of parental care, such as feeding effort) or whether they have to make the best of bad job by being less efficient at incubation (Table 1).

Here, we have shown differential parental investment of females during incubation, which can be distinguished from compensation for a reduction in parental investment by an attractive mate. From the current study, we were not able to determine whether differential allocation or differential access could explain the observed differential parental investment patterns, which requires experimental manipulation of male attractiveness (Sheldon 2000). However, whichever is the case, differential incubation investment is considered to be important in this species as a means of post-mating sexual selection (cf. Møller $\&$ Thornhill 1998). Through high total nest attentiveness (Table 1), attractive males would gain reproductive advantages in terms of increased hatching success (Gorman et al. 2005) or a reduced incubation period (Fig. 3; Voss et al. 2008), which in turn reduces the probability of nest predation.

\section{ACKNOWLEDGMENTS}

We are grateful to the residents of Joetsu City for their kind support and assistance. We also thank the members of the Laboratory of Animal Ecology of Joetsu University of Education and the Laboratory of Conservation Ecology of University of Tsukuba.

\section{REFERENCES}

Burley N (1986) Sexual selection for aesthetic traits in species with biparental care. Am Nat 127: 415-445.

Burley N (1988) The differential-allocation hypothesis: an experimental test. Am Nat 132: 611-628.

Dreiss AN, Navarro C, de Lope F \& Møller AP (2008) Digit ratios, secondary sexual characters and condition in barn swallows Hirundo rustica. Behav Ecol 19: $16-21$.

Foley JD \& van Dam A (1984) Intensity and color. In: Fundamentals of Interactive Computer Graphics. pp 593-622. Addison-Wesley, Philippines.

Gorman HE \& Nager RG (2004) Prenatal developmental conditions have long-term effects on offspring fecundity. Proc R Soc Lond B 271: 1923-1928.

Gorman HE, Arnold KE \& Nager RG (2005) Incubation effort in relation to male attractiveness in zebra finches Taeniopygia guttata. J Avian Biol 36: 413420.

Harris WE \& Uller T (2009) Reproductive investment when mate quality varies: differential allocation versus reproductive compensation. Phil Trans R Soc Lond B 364: 1039-1048.

Hasegawa M, Arai E, Watanabe M \& Nakamura M (2008) Methods for correcting plumage color fading in the Barn Swallow. Ornithol Sci 7: 117-122.

Hasegawa M, Arai E, Watanabe M \& Nakamura M (2010) Mating advantage of multiple male ornaments in the Barn Swallow Hirundo rustica gutturalis. 


\section{HASEGAWA et al.}

Ornithol Sci 9: 141-148.

Horváthová T, Nakagawa S \& Uller T (2011) Strategic female reproductive investment in response to male attractiveness in birds. Proc R Soc Lond B in press. doi: $10.1098 / \mathrm{rspb} .2011 .0663$.

Houston AI, Székely T \& McNamara JM (2005) Conflict between parents over care. Trends Ecol Evol 20: $33-38$.

Johnsen A, Delhey K, Schlicht E, Peters A \& Kempenaers B (2005) Male sexual attractiveness and parental effort in blue tits: a test of the differential allocation hypothesis. Anim Behav 70: 877-888.

Kojima W, Kitamura W, Kitajima S, Ito Y, Ueda K, Fujita G \& Higuchi H (2009) Female barn swallows gain indirect but not direct benefits through social mate choice. Ethology 115: 939-947.

Kose M \& Møller AP (1999) Sexual selection, feather breakage and parasites: the importance of white spots in the tail of the barn swallow. Behav Ecol Sociobiol 45: 430-436.

Kose M, Mänd R \& Møller AP (1999) Sexual selection for white tail spots in the barn swallows in relation to habitat choice by feather lice. Anim Behav 58: $1201-$ 1205.

Lande R \& Arnold SJ (1983) The measurement of selection on correlated characters. Evolution 37: $1210-1226$.

Lendvai AZ, Kis J, Szekely T \& Cuthil IC (2004) An investigation of mate choice based on manipulation of multiple ornaments in Kentish plovers. Anim Behav 67: 703-709.

de Lope F \& Møller AP (1993) Female reproductive effort depends on the degree of ornamentation of their mates. Evolution 47: 1152-1160.

Magrath MJL \& Komdeur J (2003) Is male care compromised by additional mating opportunity? Trends Ecol Evol 18: 424-429.

Mitchell DP, Dunn PO, Whittingham LA \& FreemanGallant CR (2007) Attractive males provide less parental care in two populations of the common yellowthroat. Anim Behav 73: 165-170.

Møller AP (1988) Female choice selects for male sexual tail ornaments in the monogamous swallow. Nature 332: 640-642.

Møller AP (1994) Sexual Selection and the Barn Swallow. Oxford University Press, Oxford.

Møller AP \& Thornhill R (1998) Male parental care, differential parental investment by females and sexual selection. Anim Behav 55: 1507-1515.
Nakamura N \& Nakamura M (1993) Genshoku nihon yachou seitai zukan-rikuchouhen. Hoikusha, Tokyo (in Japanese).

Neuman CR, Safran RJ \& Lovette IJ (2007) Male tail streamer length does not predict apparent or genetic reproductive success in North American barn swallows Hirundo rustica erythrogaster. J Avian Biol 38: 28-36.

Ninni P (2003) Carotenoid Signals in Barn Swallows. $\mathrm{PhD}$ thesis, Université Pierre et Marie Curie, Paris.

R Development Core Team (2008) R: A language and environment for statistical computing. R Foundation for Statistical Computing, Vienna. ISBN 3-90005107-0, URL http://www.R-project.org.

Safran RJ \& McGraw KJ (2004) Plumage coloration, not length or symmetry of tail-streamers, is a sexually selected trait in North American barn swallows. Behav Ecol 15: 455-461.

Safran RJ, Pilz KM, McGraw KJ, Correa SM \& Schwabl H (2008) Are yolk androgens and carotenoids in barn swallow eggs related to parental quality? Behav Ecol Sociobiol 62: 427-438.

Sanz JJ (2001) Experimentally reduced male attractiveness increases parental care in the pied flycatcher Ficedula hypoleuca. Behav Ecol 12: 171-176.

Sheldon BC (2000) Differential allocation: tests, mechanisms and implications. Trends Ecol Evol 15: 397402.

Smith HG \& Montgomerie R (1992) Male incubation in barn swallows: the influence of nest temperature and sexual selection. Condor 94: 750-759.

Tajima K \& Nakamura M (2003) Response to manipulation of partner contribution: a handicapping experiment in the Barn Swallow. Ornithol Sci 2: 65-72.

Tinbergen JM \& Williams JB (2002) Energetics of incubation. In: Deeming DC (ed) Avian Incubation. pp299-313. Oxford University Press, Oxford.

Turner AK (2006) The Barn Swallow. T \& A D Poyser, London.

Václav R \& Hoi H (2002) Deferent reproductive tactics in house sparrows signaled by badge size: Is there a benefit to being average? Ethology 108: 569-582.

Voss MA, Rutter MA, Zimmerman NG \& Moll KM (2008) Adaptive value of thermally inefficient male incubation in barn swallows (Hirundo rustica). Auk 125: $637-642$.

Witte K (1995) The differential-allocation hypothesis: does the evidence support it? Evolution 49: 12891290 . 